\title{
Model of Self-organizing Knowledge Representation and Organizational Knowledge Transformation
}

\author{
Oleg Vasylovych Moroz \\ Software Engineering Department, Faculty of Cybersecurity, Computer and Software Engineering, National Aviation University, Kyiv, \\ Ukraine
}

\author{
Email address: \\ omoroz@nau.edu.ua
}

\section{To cite this article:}

Oleg Vasylovych Moroz. Model of Self-organizing Knowledge Representation and Organizational Knowledge Transformation. American Journal of Artificial Intelligence. Vol. 4, No. 1, 2020, pp. 1-19. doi: 10.11648/j.ajai.20200401.11

Received: February 24, 2020; Accepted: March 16, 2020; Published: March 24, 2020

\begin{abstract}
The purpose of the paper is development of a conceptual model for the representation of knowledge as an active intellectual substance and, on this basis, study of metaphysics of knowledge transformation process being produced both individually and collectively in the practice of organizations. The first principle of knowledge engineering, as Edward Albert Feigenbaum noted, says that the power in solving problems that an intellectual subject (person or machine) manifests in the process of activity depends primarily on its knowledge base, and only secondly on the methods of inference used. Strength is hidden in knowledge. The process of producing knowledge is permanent and does not depend on whether an individual is going to use this knowledge or not. Knowledge constantly produces new knowledge regardless of the owner's desire. Besides that, knowledge can't arise from nothing, but always - from some knowledge obtained earlier. As well as the intelligence, knowledge is an emergent instance arising from the collective interaction of a lot of intellectual atomic elements of knowledge (knowledge quanta). Idiosyncrasy of this interaction is expressed precisely in the creation of new knowledge. Due to postulating the knowledge self-organizing, the hierarchical knowledge structures in memory and the process of thinking as a kind of syntax for the procedure of new knowledge generation are described. This is an effort towards understanding the memory mechanisms, the process of thinking, the sources of heuristic knowledge just through the inner nature of knowledge. Also, based on the knowledge self-organization principle, an archetype of the appropriate knowledge-based system architecture is presented too. As an implementation of the concept, the perceptual act model is described, and on its base, a possible scenario for the behavior of a robot meeting an obstacle in its path is considered. As the mutual transformation of tacit and explicit knowledge makes new knowledge, the impact of the self-organization of knowledge on the transformation process as well as conditions of self-organization of both individual knowledge and organizational knowledge are analyzed in detail. Finally, modification of the known model of knowledge dimensions by Nonaka and Takeuchi is proposed. Because of the native activity of knowledge, it is impossible to build a knowledge management system without considering the internal structure of knowledge and its emergent ability to self-organize. Ensuring the natural process of knowledge development at all ontological levels in an organization is an essential prerequisite for the evolution of values in this organization.
\end{abstract}

Keywords: Knowledge Representation Model, Knowledge Self-organizing, Knowledge Management, Knowledge Transformation, Model of Knowledge Dimensions

\section{Introduction}

The root issues of knowledge management (KM) are closely related to the fundamental problems of artificial intelligence. Traditionally, the awareness of artificial intelligence is considered in parallel with the problem of determining intelligence in general. Since such a definition does not exist so far, the natural question arises - why? One of the key signs of the presence of intelligence is the human's ability to self-awareness, self-analysis. But does the human have the necessary knowledge to be able to cognize himself?

The topics such as the nature of memory of living beings, the essence of the process of thinking or the source of heuristic knowledge are often discussed yet rarely understood. Nevertheless, without a clear understanding of these categories, it is impossible to develop an adequate $\mathrm{KM}$ 
framework. What do we know detail about our own brain, in addition to the fact that its various areas are responsible for one or another type of activity? Despite this, approach to the development of digital computers by analogy with the functioning of the human brain seems to be the most promising, and "this is a representation based on common sense." [1]

As for the process of thinking, it is traditionally emulated by formal-logical output schemes implemented in a physical symbol system. Newell-Simon hypothesis states that the system "has the necessary and sufficient means of general intelligent action", but it goes without saying that intellectual behavior is not just about symbolic calculations. How can symbolic calculations convey emotions and feelings, or how to represent the diverse relationships between real-world objects such as modality, idiomaticity or emergence? This approach is a manifestation of classical rationalism and has been repeatedly criticized, although symbolic systems dominate in studies of artificial intelligence up to nowadays. And this criticism is also a representation based on common sense, according to which the world around us is inherently more irrational than rational. Human behavior is mostly irrational and is controlled by the subconscious. According to the estimates of psychologists, as much as $90 \%$ of the decisions we make in life are irrational. It is through irrational behavior that organisms evolve, so in the notion of intelligence emphasis should not be on the ability to handle logical statements about the world, but on the ability to ensure survival and development in this world. Therefore, any development of an artificial intelligence system must be based on two basic themes: "first, the view of intelligence as rooted in culture and society and, therefore, emergent. The second theme is that intelligence is reflected by the collective behaviors of large numbers of very simple interacting, semi-autonomous individuals, or agents. Whether we take these agents to be neural cells, individual members of a species, or a single person in a society, their interaction produce intelligence." [2] The key term here is "agent interaction". Undoubtedly, the essence of such interaction lies in the exchange of information and knowledge as well as, possibly, energy. Consequently, the problem of knowledge representation, knowledge's emergence, development and transfer will be the key one for any approach to artificial intelligence modelling. Thus, knowledge should be regarded as the foundation of consciousness, thinking and ultimately intellect. This is our main working hypothesis.

The prerequisite for the existence of all living beings is their evolution. In the biological world, mutations give impetus to evolution that generate competition between individuals and species. For animals, there is only one type of knowledge - behavioral knowledge, that is knowing how to behave in the environment in order to survive. In the world of people, there are also social and economic relations, the latter being dominant. The role of "mutations" for the human community is played by technologies that are knowledge of how to produce products better and more efficiently than competitors. Such knowledge can and should be managed, such matter in the era of globalization and universal digitizing computer Knowledge Management Systems have appeared. However, in order to manage knowledge in practical activities, it is needed to know how new knowledge arises, how and why they change, where and in what form they are stored, how to extract them as necessary, how to transfer and use them with maximum efficiency.

So, to determine the intellect the necessary intellectual technologies are to be developed. Postulating the intellect as an emergent means of collective adaptation in the environment, this problem can be formulated as follows: is it possible to accumulate knowledge and manipulate them in the same way as community intelligent beings do? In the article, we intend to consider two key moments in the context: knowledge representation model and metaphysics of the transformation of knowledge collectively produced and used in people's practice.

\section{Literature Review}

\subsection{Artificial Intelligence Techniques}

Among the Artificial Intelligence (AI) techniques [3, 4] developed up today one can distinguish four basic types:

1. Artificial Neural Networks (ANN).

2. Genetic Algorithms (GA).

3. Multi-agent Systems (MAS).

4. Hybrid systems (combinations of previous and some others, such as fuzzy-logic or probabilistic reasoning).

They allow implementing individual cognitive abilities such as pattern recognition, translation from natural languages, diagnosis of diseases, and others. This is the so-called narrow artificial intelligence". On the other hand, there is the problem of integrating various intellectual abilities, known as "artificial general intelligence" [5]. Combining some of soft computing methods [6] seems could solve this problem, nevertheless, most hybrid systems are based on ANN and aimed at improving their learning ability with all their inherent disadvantages. Actually the successes of AI development are mainly associated with the machine learning methods of deep learning and reinforcement-learning based on ANN (ANN/ML), but the most advanced direction in the field of AI seems to be the Evolutionary Artificial Neural Networks (EANN) [7, 8, 9], in which neural networks are trained using genetic algorithms and are even able to evolve their architecture.

Despite the certain successes achieved, all these approaches, including hybrid systems, have one common drawback being not able to classify ad hoc knowledge, which means that their adaptive abilities are unsatisfactory, and, in addition, the mechanisms on which intelligent systems make conclusions are far from the natural process of thinking, although they try to imitate it. Each of the mentioned approaches focuses on a certain aspect of the phenomenon of intellectual behaviour, in other words, they are narrowly focused in terms of the versatility of the manifestations of natural intelligence. Let's briefly consider the advantages and disadvantages of these 
approaches in the context of the earlier comments made regarding the epistemology of AI.

Artificial Neural Networks

By far, neurocomputing is the most dynamically developing approach for creating knowledge-based systems. With all the advantages and achievements, ANN clearly lack "intelligence" due to the primitive synthesis of knowledge. They really are a parody of how the brain processes information in the truest sense of the word. The ANN's thinking mechanism is a "black box", however, just like the real brain (in this they are similar indeed). Yes, ANN can be trained, but they do not may to synthesize knowledge from previously acquired knowledge. In addition, they need a huge amount of digitized information for training, and although the volume of such data is constantly growing, the question "How many cats will it need to identify a cat?" still has an ironic connotation regarding the intellectual abilities of ANN. Moreover, the problems of "catastrophic forgetfulness" and the "black box" create a precedent for the next AI winter rather than a breakthrough in the creating of artificial general intelligence.

Indeed, with the help of ANN/ML, the problem of pattern recognition is quite successfully solved $[10,11]$, although the neural network is easy to fool [12]. However, pattern recognition alone does not produce anything without storing these patterns in memory for further quick recognition or, moreover, recognition by partial (truncated) features. It is obvious that this requires a suitable model of knowledge representation, which would provide an autonomous quick search for relevant information in memory and use it to obtain new knowledge.

Genetic algorithms

GA can be used both separately as heuristic methods for solving discrete (including multi-purpose) optimization problems [13-15], and for the training of neural networks [16-20] and even interpreting the results of their work [21]. And, although when choosing between the semantic approach and GA, the latter is given to preference more often, the main drawback of GA is that genetic operators (operators of mutation, selection, crossbreeding) lack semantic content. Inheriting the rule methodology and the neighborhood structure from the Cellular Automata, GA are unnecessarily mechanistic and abstract like the Laplace demon, who is capable of knowing the position and speed of each particle in the Universe to see its evolution both in the future and in the past. Unlike real evolutionary-genetic transformations, GA does not solve the problem of the "survival" of the population as a whole and are aimed at optimizing certain moments in its vital activity, but "... it would be rash to see in optimization the key to understanding how populations and individuals survive" [22]. Both GA and EANN can be quite effective for solving differential equations (including evolutionary ones) and systems of such equations [23-26]. However, using evolutionary computational methods, it is impossible to predict abrupt transitions in the evolution of a complex open system from a state of chaos to a qualitatively new state, since genetic operators alone are not enough to model evolution understanding the nature of the collective forces of interaction between "individuals" that lead to irreversible and indeterminacy of the phase trajectories of such systems is needed. Meanwhile, such behavior is characteristic of evolving and self-organizing open systems, among which, no doubt, biological and social systems are.

Multi-agent systems

MAS is composed of intelligent agents that actively interact with each other and with the environment. Unlike ANN, in which the interaction of neurons is modeled by a variety of mathematical equations, MAS agents are autonomous, therefore, usually one or more agents are selected that model the network of connections between agents. Functioning each according to its own program, altogether the agents create the context of their collective intellectual interaction, which is usually called Swarm Intelligence (SI) [27, 28]. Naturally, in such a system the coordination control problem (orchestration) arises. There are a lot of models [29] of the orchestration in MAS and some approaches for solving the problem have been proposed too, among them are follows: event-triggered distributed model predictive control approach [30], graph-theory-based approach [31] and holon-based approach, being used in the holonic multi-agent systems [32-35], which provide self-organizing and evolution mechanism but unfortunately have no the ability to represent and use corporate knowledge as well as to allow the user to enter new knowledge "on the go". In this regard, an approach [36], which generally no mechanisms for coordinating the actions of agents are in and for which just the variety of actions is important, looks more attractive. The result of such a simulation of collective decision-making is obtained as the value averaged over the ensemble, but naturally, some questions are arising in that regarding the averaging technique. In the more common case, each agent may be represented as the set of some unified knowledge units or "hypothesis" [37], each of which is simple and interpretable, what one gives the opportunity to unite different kinds of knowledge and to use generalized algorithms for knowledge processing.

Considering the growth over the past decade the tendency to combine ANN and evolutionary optimization methodologies, let us imagine a hypothetical hybrid system consisting of intelligent agents with the ability to deep learning and specializing each in its narrow cognitive domain. Each of the agents, therefore, is an expert in their field, which is consistent with the distribution of functions between control centers in the brain. However, for such a system to be able to make reasonable decisions, it must be capable of deductive and inductive thinking based on individual knowledge of agents. This problem, in principle, can be solved in two ways: either by highlighting additional agents that would categorize knowledge using the knowledge otology and connect the necessary agents to the solution of the problem, or by endowing all agents with the ability to independently search for the necessary relevant knowledge in the system, which, in fact, is a self-organization of knowledge. Such an understanding of the self-organization of knowledge is closely related to the concept of emergent intelligence, which arises spontaneously in the society consisting of individual simple 
agents that are fundamental thinking beings [38]. Thus, it can be argued that the emergent self-organization of knowledge is just the prerequisite for the creation of collective intelligence. Moreover, the self-organization of knowledge should be considered as a characteristic property of artificial general intelligence systems.

\subsection{Knowledge Representation Models}

Regardless of the technique used, any implementation of artificial intelligence is impossible without a suitable model of knowledge representation, which would ensure the effective storage of knowledge in memory and its manipulation in the thought process. To date, many models of knowledge representation, based on the study of the principles of the organization of human memory and cognitive psychology, have been proposed. The most famous ones are the following:

1) Model of production rules, in which knowledge is presented in the form of rules "IF < condition $>$ TO $<$ action>". With many rules, difficulties arise due to the inconsistency of the rules, but the main problem is that the expert's knowledge is not always possible to represent as a set of rules, not to mention formalizing them. Due to the complexity of changes and the poor scalability of production rules, their scope is limited to specialized diagnostic expert systems, which nevertheless continue to be quite popular [39], and, in particular, hybrid expert systems combining the strengths of neural networks and production rules that allow to operate incomplete and inaccurate data [40-42].

2) Network model (semantic networks), in which knowledge is represented in the form of graphs [43]. The key problem for this model is that in order to obtain a conclusion based on the semantic network, the corresponding ontology of the subject area is necessary. Ontologies based on descriptive logics are the most advanced way of representing knowledge and used where high accuracy of recognition of the semantic relations between objects of the real world is required. However, this is possible only for a specific subject area, since the number of distinguishable concepts in this world is infinite. For this reason, the idea of implementing the Semantic Web [44] as a publicly accessible global semantic network has not yet been realized and can hardly count on success. Both the limits of applicability of ontologies and the adequacy of their application to represent knowledge provide the basis for numerous debates [45]. Most corresponding to modern concepts of human memory organization, semantic networks, however, are faced with the intractable problems such as finding solutions, use, and modification of knowledge with an increasing number of concepts and relations between them in the case of a real complex systems.

3) Minsky Frame model [46], in which knowledge is presented in the form of frames with two types of slots system and user-defined. The hierarchical structures of frames are well suited for representing taxonomies, but their application is limited by the relatively high complexity of introducing changes to an already created hierarchy. Like semantic networks, frame-based networks are passive structures of knowledge, that is, their languages of knowledge representation do not contain inference mechanisms, therefore, they require integration with other means of knowledge processing, for example, with production rules [47] or logic programming [48].

4) Neural network model, in which knowledge is represented as the sets of synaptic coefficients, getting their values in the process of ANN training with an explicit or implicit teacher (supervised learning), which controls learning by changing the network parameters and, possibly, the network topology, or without it (unsupervised learning). Despite the numerous applications of ANN using supervised learning for solving problems of classification, clustering, and even categorization, it is obviously more natural to train a neural network without a teacher, when the network independently forms the structure of its knowledge by trial and error (such networks are called self-organizing). The most well-known (but not the only) kind of unsupervised learning model is Kohonen Self-Organizing Maps (SOM) [49]. As a result of its training, SOM breaks multidimensional input data into clusters and visually maps them to a two-dimensional grid, but, unlike the semantic network, which patently reflects the semantic relations between concepts of the subject area, SOM allows only to evaluate the semantic closeness (or distance) between the concept-clusters. In addition to the difficulty of semantic interpretation of the network topology and the linking of map's somites with specific concepts from the subject area, it should also be noted the difficulties of visualization and interpretation of codebooks with increasing dimensionality of input data, as well as the dependence of the results on the initial network parameters. It is also necessary to clarify the meaning of the term "self-organization" as applied to ANN using unsupervised learning. Here, self-organization is understood as the possibility of learning without a teacher, that is, in fact, continual self-organization, which involves an a priori setting of the initial network parameters by the experimenter, in contrast to the emergent coherent self-organization that inherent in open systems with a large number of reflectively interacting elements, which are constantly subject to the influence of the collective forces of interaction that they themselves create. In principle, SOM can exhibit coherent phenomena, but additional methods, such as U-matrix [50], are required to visualize it.

A complete analysis of the problem of knowledge representation is beyond the scope of this article; therefore, we restrict ourselves to stating the common weaknesses for all the above models of knowledge representation, especially since they are well known. 
The first drawback is the focus on the presentation of primarily declarative knowledge. The conditional division of knowledge into declarative and procedural, originating from the Newell-Simon problem solver [51], is not justified, since they are always inseparably linked with each other, and the problem is not that there is no universal logical inference mechanism, but that it has not to be built on the basis of formal logic, but on the basis of more general Kant's transcendental logic. The only model of knowledge representation in which declarative and procedural pieces of knowledge are not torn off from each other is the frame model, but it obviously oversimplifies the true state of things. For this reason, many combined ways of knowledge representing have been developed [52], based mainly on the KL-ONE/KL-TWO formalism [53]. And in the end, it is completely incomprehensible what to do with the knowledge which "may be represented in propositions, more of it in imagelike forms, and the rest of it inhabits, vague intuitions, and "gut feelings" that are never verbalized or visualized.... Its fluid, heterogeneous, ever changing, and often inconsistent nature could be better characterized as knowledge soup." [54]

The second drawback is a consequence of the first one and consists in the fact that they do not ensure the unity and collective integrity of the processes that together form the consciousness, since the thinking process is practically replaced by exhaustive search of production rules, analysis of the topology of semantic or frame networks or precedents considered in the learning process, after which, as a rule, follows the interpretation of the results and determination of the degree of their reliability. In other words, in the process of thinking the leading role is assigned to the inference procedure, and knowledge plays a secondary role. This is wrong since the inference mechanism should really be universal and applicable in any situation, but the result depends, first of all, on the knowledge that is involved in this situations, that is knowledge should be primary, and for this, they should not be in a passive form. Moreover, with such an abundance of hybrid models of knowledge representation and their focus on certain types of knowledge, one does not have to talk about any possibility of creating an integrated way of perceiving the surrounding reality - this is simply unrealistic.

And finally, the third drawback is the lack of a mechanism to support the self-organization of knowledge. It is possible to represent the memory structure in the form of interconnected ontologies or frames or at least self-organizing maps, but the self-organization of knowledge will be imaginary, since knowledge is in a passive form and the complexity of the attached procedures for their processing will be polynomial only in the particular case of a certain subject area, and in the case of self-organizing neural networks this is only the possibility of learning without a teacher, but just not the internal state of knowledge itself, correlated with a certain system of concepts or categories.

The knowledge representation model proposed in this article addresses these shortcomings. But before proceeding to the consideration of the model, we single out several key requirements that the artificial general intelligence system should satisfy in our opinion, considering the advantages and disadvantages of the AI methodologies presented above:

a) Adaptability to changing environmental conditions, scalability, and flexibility of the architecture.

b) Learnability, including through knowledge transfer.

c) Implementation of the mechanism of the thinking process based on the native ability of knowledge to be self-organized and create new knowledge instead of a logical conclusion or analysis of precedents.

d) Self-organization of knowledge in memory as a result of the collective interaction of a lot of simple structural elements of knowledge with the ability to perform a targeted search in the corresponding memory structures.

\subsection{Knowledge Management}

The subject of KM has been widely presented in publications since the 1990s. "The overall volume of scient metric knowledge management works has been growing..., but their key findings are somewhat inconsistent. Most scient metric knowledge management research is published in non-knowledge management-centric journals. The knowledge management discipline has deep historical roots. It suffers from a high degree of over-differentiation and is represented by dissimilar research streams." [55] The trend is obvious the studies are distributed in many ways, which, in turn, calls for the development of special normative classification schemes [56]. In reference [56] seven categories of studies, each of which is also broken down into some subcategories, are allocated: Knowledge and KM artefacts, KM frameworks and models, KM systems, Ecosystem, Influencing factors, KM reference disciplines, Research design and research method.

On the one hand, this speaks about the growing interest of researchers in the discipline of KM, which affects a great many of the fundamental and applied problems. On the other hand, there is clearly a lack of a common understanding of the phenomenon of knowledge proper as well as a unified approach to the notion of "knowledge management". There are two historically and territorially developed approaches to KM in the organization: the "Western" approach and the "Japanese" approach. The "Western" approach is characterized by an emphasis on the processing of explicit knowledge based on information technology and systems. The "Japanese" approach is characterized by the organization is to be viewed as a living organism with memory and knowledge being created on the basis of subjective, individual tacit knowledge. Common for both approaches feature is that KM must have an impact both on organizational effectiveness and innovations. However, many researchers note the primacy of innovation in assessing the role of KM in organizations. In the context of innovation, the main benefit of $\mathrm{KM}$ is the socialization of individual knowledge. This process does not only promote the dissemination of knowledge in the organization, but it is also a prerequisite for the generation of new knowledge. The main obstacle to the socialization of individual knowledge is the cultivation of survival relationships in the organization when employees are 
competitors, not associates. In such conditions, the employees obviously will not have the desire to share knowledge and the whole sense of KM is lost.

Contrariwise, always keep in mind that organizational knowledge is the product of collective intellectual interaction of its employees aimed to achieve common goals and to affirm common values and beliefs. If the organization is a single organism where everything relates to everything, it seems reasonable to develop principles and methods of KM within the framework of the well-known concept "7-S Framework McKinsey". This concept is implied the management infrastructure of an organization is represented as a set of seven interrelated elements: Shared values (or Superordinate goals), Strategy, Structure, Systems, Staff, Style, Skills. The peculiarity of the 7-S model is its shared-values-centered, so all other elements "rotate" in the field of attraction of these goals, looking like planets in their orbits.

Most research in KM refers to developing KM frameworks. Practically all KM frameworks presented in the literature can be divided into two types [57]: Broad KM Frameworks and Specific KM Frameworks. Common for all Broad Frameworks is the description of the KM process as a set of knowledge manipulation procedures (or functions), such as creation, manifestation, use, and transfer. This approach to developing KM frameworks can be called a functional or descriptive one. An alternative to the descriptive approach would obviously be an approach based on a detailed analysis of the logic of the interrelationships of the elements in the 7-S model in the context of KM and knowledge representation as an interactive resource of the organization.

Unlike Broad KM Frameworks, which cover the overall KM discipline, Specific KM Frameworks focus on specific issues of $\mathrm{KM}$ theory, such as the life cycle of knowledge, technologies used, categorization and interpretation of knowledge, perspectives of KM as an organizational asset and others.

One of the most well-known models of the life cycle of organizational knowledge is the epistemological scheme of Nonaka and Takeuchi [58], based on the representation of the process of organizational knowledge creation in the form of a spiral process of transformation of tacit (cannot be formalized) and explicit (can be formalized) knowledge. Knowing as "spiral of knowledge creation", this model considers four ways of the knowledge transformation: from tacit to tacit (Socialization), from tacit to explicit (Externalization), from explicit to explicit (Combination), from explicit to tacit (Internalization), which all together form the acronym SECI, so the model is named as the SECI Model too.

Certainly, studies of the structure of organizational knowledge affecting concepts such as organizational memory, organizational wisdom or mind deserve special attention. Obviously, in this case, we are talking about collective knowledge and the collective mind, although researchers of such concepts, as a rule, use terms and concepts of cognitive psychology and limit themselves only to one of elements of 7-S Framework such as strategic management [59]. At the level of the organization, the term "knowledge structure" refers to shared values and beliefs [60]. Common values and beliefs, as well as the culture of relationships in the organization, do not develop quickly - it takes years. During this time the organization is learned, and the accumulated knowledge "settles down" in the form of business strategy and business infrastructure, skills and abilities of employees, organizational structure, the structure of information and technical systems, management styles. Today, arguments in support of the message that organizations have additional "reservoirs" of memory and knowledge, apart from the individual brains of their employees, are more than enough, but still, such concepts seem to remain the objects of a few predominantly theoretical studies.

\section{Knowledge Representation Model}

\subsection{Extracting Knowledge from Data}

Knowledge can obviously be derived from data, but for this, data must overcome a certain threshold - the line of knowledge, which demonstrates yet another manifestation of the dialectical law of the transition of quantity to quality. The implicit knowledge contained in the data can be extracted with the help of various analysis methods, but they all have some common drawbacks due to the obvious fact that the data is highly susceptible to change. For the analysis, the data must first be structured, that is, the hidden entities must reveal, their properties and characteristics, as well as the relationships between these entities. Data can be modified as a result of both the interaction of the original entities and the evolutionary development of these entities themselves.

Knowledge is extracted from the data by synthesis. The process of synthesizing knowledge from data occurs by establishing the correspondence of the basic (or key) characteristics of the data and is like assembling puzzles. The selection of the basic characteristics of the data is equivalent to their categorization and is based on the method of correspondence. The essence of this method lies in the fact that the basic characteristics of data can be identical to the reference set of characteristics (RSC), which are primary (initial) concepts, from which more complex derivative concepts of the whole set of concepts of the domain, that is conceptual apparatus, are formed. Therefore, the first step in building a KM system is to create an ontology of the related knowledge.

From the point of view of managing actions in interaction with the environment, knowledge will always be primary for a person, whereas data will be secondary. A person receives knowledge from his life experience, constantly perceiving the data coming from the surrounding environment and relying on that primary knowledge received from his parents in his childhood.

Let's consider the most common situation that every person in his life could observe more than once. Let's imagine a glade where children play. They carry toys from one part of the glade to another, without thinking where these toys came from. They compare the visual images of toys with their desires and 
thus choose the toys they like. So, children use their knowledge, but the toys can evoke new desires of the children, creating an aspiration for new knowledge. Playing, children use their knowledge to control their behavior. They also use the data in the game, picking out toys, for example, by color, shape, texture, but they do not use data to organize the game. To make the game they need knowledge because the game is a certain chain of events controlled by the reaction of players in accordance with certain rules.

The knowledge used by people in their activities is closely related to the processes and operations executed by them in these processes. In his professional work, as well as in any other, a person does not analyze the data, but he synthesizes the knowledge necessary to perform various actions on the way to achieving the goal. Certainly, a person extracts knowledge based on his own life experience, but firstly he analyses neither facts nor events or phenomena, he simply assesses all the happening around him relying on existing life experience. In other words, a person "gets used to" the surrounding reality primarily and only then begins the analysis followed by the synthesis of new knowledge based on both the results of the analysis and already existing knowledge.

\subsection{Quanta and Clusters of Knowledge}

As while creating any other model, one cannot do without postulates:

Postulate 1: Knowledge in the brain is quantized.

Postulate 2: Knowledge quanta can form clusters, creating new knowledge.

Postulate 3: Knowledge quanta have activity being capable to recognize one another.

Knowledge quanta have some characteristic properties:

1. If one compares knowledge in general with a forest in which there are many fir trees with fir-cones, then the knowledge quanta are the fir-cones. These "fir-cones" can grow, ripen, give seeds, from which later new knowledge can grow, and fall (disappear).

2. They can interact with each other. This interaction is due to collective effects, the nature of which is akin to those that cause birds to gather in flocks and control these flocks as a single living organism.

3. Knowledge quanta are wave packets, characterized by a certain polarization value and a set of characteristic frequencies. The specific set of the characteristic frequencies values determines the RSC, that is, it corresponds to a certain concept of the domain. Each $\mathrm{RSC}$ is knowledge quantum, but not all knowledge quanta are RSC.

4. Knowledge quanta can be associated and form new RSCs.

Knowledge quanta can form clusters in the memory, but these clusters are virtual. It means that knowledge quanta can form virtual counterparts which clusters are composed of, at that each quantum of knowledge can create his counterparts arbitrarily much. Clusters are formed from knowledge quanta when one or more characteristic frequencies coincide. Note that neurons in the brain form clusters in accordance with the same principle, that is, by the coincidence of the frequencies of the transmitted signals.

A cluster can consist of one quantum if this quantum is an RSC. A quantum that is not an RSC is simply a fact. The facts form the shell of the cluster (husk), which, unlike the cluster, is unstable - the facts can be easily added to the shell and removed from it. The husk consists of knowledge quanta which are different from those contained in the cluster by the special status of the "associated member". These agglomerates of knowledge quanta correspond to the knowledge stored in the so-called short-term memory. The clusters themselves correspond to the knowledge stored in the so-called long-term memory.

In terms of the coupling strength between knowledge quanta, clusters consisting of these ones have a fairly "dense" core and the "looser" periphery. Knowledge quanta which comprise to the core of cluster have two or more coincident characteristic frequencies and form the elements of the semantic core of a certain domain. The knowledge represented by the periphery of the cluster represents refinements, extensions and additions to this semantic core.

To sum up then all knowledge is divided into RSC and concepts, which derived from RSC, and is represented in memory in the form of clusters, covered with husks of facts.

\subsection{Knowledge Structures in Memory}

In memory, knowledge is formed by a tree-like hierarchical structure, which we will call as the knowledge tree. The knowledge tree consists of five backbone subtrees, in each one the nodes are located on three levels (Figure 1). General management is provided by a control center of knowledge tree, which includes five controllers managing subtrees. Similarly, each of the nodes lying on the 1st, 2nd and 3rd level has five child nodes and, accordingly, five controllers. Each of these controllers directly manages its child node (is "owner" of the node), but still has access to the other four child nodes. The leaf nodes that are children of the third-level nodes have no subordinate nodes and therefore have no controllers.

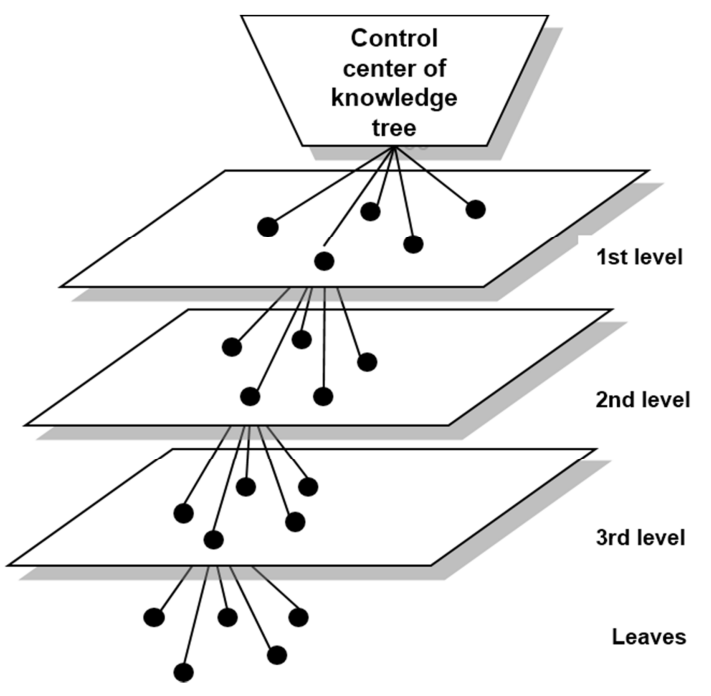

Figure 1. Structure of the knowledge tree (fragment). 
Direct interaction is possible only between nodes that have a common ancestor. This means that within each of the five backbone subtrees, the nodes can interact directly, that is, over the network data model. Interaction between the nodes of different backbone subtrees is carried out by migration of copies of the node from one subtree to another, and a copy of such node is marked as "alien" in that subtree which this node migrates into.

Each node in the knowledge tree is a container in which clusters of knowledge quanta are stored. Each quantum has five characteristic frequencies, each of which corresponds to one of the five backbone subtrees. If a knowledge quantum falls into one of these subtrees, the exact frequency that corresponds to that subtree is excited. In addition, knowledge quanta contain a bit map of the entire system of nodes of the knowledge tree, that allows to quickly determine which node the given quantum belongs to.

Knowledge possess activity, and this in principle distinguishes them from data. The process of producing knowledge is permanent and does not depend on whether a person is going to use this knowledge or not. Knowledge constantly produces new knowledge regardless of the person's desire. To do this, the control center of knowledge tree should work with a certain clock frequency and issue commands from a specific set of commands. The total number of commands in this set and the number of instructions executed in a single cycle are the most important characteristics of the control center of the knowledge tree. The main commands are as follows: searching for, adding, removing, duplicating, clustering (association) of knowledge quanta.

Knowledge can't arise from nothing. The source of knowledge is always some knowledge obtained earlier. The tree of knowledge subsequently grows based on primary knowledge, which transmitted from parents in infancy for everyone. This process is gradual. First, the child is given behavioral knowledge: how to eat, how to walk, how to dress, how to behave with other people, and others. Then verbal knowledge is already transmitted. Animals transmit to their cubs only behavioral knowledge. When knowledge is passed on to the child, it is not indicated where the knowledge should be placed in the knowledge tree - the child himself "introduces" it into his KM system.

The human's KM system is autonomous absolutely and does not depend on any systems. To function properly it only needs to be recharged with the energy necessary to operate the clock frequency generator of the knowledge tree control center.

Adding new knowledge to the knowledge tree is accompanied by the branching of the backbone subtrees. New knowledge literally grows like branches on a tree from the kidneys. "Kidneys" on the knowledge tree arise accidentally or as a result of a purposeful searching for solutions of problems or tasks. The process of adding new knowledge to the knowledge tree comes about as follows. If a new fact (a quantum of knowledge with a special status) appears, the knowledge quanta which this fact could be associated with are searched. If such quanta are found, the status of the fact is changed to normal and it is added to the found cluster, otherwise it is added to the knowledge tree as a separate quantum.

Searching for the container node for the newly added quantum occurs by the characteristic frequencies that form the vector $F(f 1, f 2, f 3, f 4, f 5)$. One of the frequencies is the activation frequency and always corresponds to a specific backbone subtree. Each node in the knowledge tree has its own fixed frequency range which is discrete and consists of five subranges. The sampling step of the frequency range is different for nodes of the 1st, 2nd, 3rd level and leaf nodes, and can vary throughout the life of individual. When a quantum is to add to the knowledge tree, the pre-processor of the knowledge tree control center determines the frequency of activation of this quantum by a certain algorithm. Knowing the activation frequency, it is easy to determine the subtree and the container node for the newly added quantum. Then the value of the activation frequency is stored to the corresponding field of the vector $\mathrm{F}$, and the status of this quantum is changed to "original" value. If the quantum is cloned later and its clone migrates to another subtree, the pre-processor of the knowledge tree control center determines the frequency of this clone for just this subtree and the frequency is stored to the corresponding field of the vector F, and the "clone" value is stored to the status field of this quantum. Any different quanta can have the same sets of characteristic frequencies, but the polarization values for them must be different.

As noted above, among the five characteristic frequencies of a knowledge quantum one is the frequency of its activation, it is from the frequency range of that backbone subtree which this quantum is contained in. The activation frequencies are integers from 1 to 1000 . This range is divided into five subranges: $1 \div 199,200 \div 399,400 \div 599,600 \div 799,800 \div$ 1000. The other four frequencies are set depending on which subcategory of knowledge the quantum falls into.

Knowledge categories form a hierarchy with 3 levels of nesting. Five basic categories of knowledge are located at the first level of this hierarchy, these are the following:

1. Behavioral knowledge (behavior in the surrounding world).

2. Verbal knowledge (communication with other people).

3. Search engine (information search).

4. Knowledge about the structure of the external environment and its place in it (how the world works).

5. Knowledge of work activity (how to do something).

Each of these basic categories is divided into 5 subcategories, which one, in turn, are also divided into 5 subcategories. For example, in the category "Behavioral knowledge", one can distinguish the following subcategories:

I.1. Treatment and disease.

I.2. The technique of pattern recognition.

I.3. Social behavior.

I.4. Behavior in extreme situations.

I.5. Life of a biological creature

The category "Knowledge about the structure of the external environment and its place in it" includes the 
following subcategories:

IV.1. Consciousness

IV.2. Microcosm

IV.3. Macrocosm

IV.4. Organic life

IV.5. The states of matter

And subcategory "Consciousness", in turn, includes the following subcategories:

IV.1.1. Perception

IV.1.2. Thinking

IV.1.3. Memory tree

IV.1.4. Feelings

IV.1.5. Emotions

The polarization vector is used for quantum clustering. The dimension of this one is five: $\mathrm{P}(\xi, \mathrm{p} 1, \mathrm{p} 2, \mathrm{p} 3, \mathrm{p} 4)$, where $\xi$ is the actual polarization value, which is a complex number and determines the direction of the polarization vector, other four elements are frequencies used to associate the quanta into clusters. The frequency elements of vector $\mathrm{P}$ are integer and have their ranges of admissible values, which are floating depending on the basic category of knowledge. These elements make sense of the relationship between quantum and cluster, which the quantum is in, for example, relations of generality, aggregation, modality, and others. These relations are divided into sub-ranges arbitrarily as the knowledge tree is filled, that is, as the individual is trained. In the same way, knowledge in the professional activity of a person is accumulated, but all the knowledge obtained in this case falls into one basic category - the fifth one.

The clusters formed in this case have a hierarchic structure with one root element (root quantum of cluster). At the same time, they are quite specific and can be called clusters only conditionally. The existence of the clusters as well as the tree of knowledge is due to the forces of collective interaction that arise at the time of the birth of a person and disappear at the time of his death. These forces are universal: they not only control human knowledge, they can create worlds, and everything that surrounds us is just part of these worlds. In fact, the knowledge tree itself is a world that is created within everyone, but it is not a reflection of the surrounded world - it is the result of the individual's "living into" this world. Everyone has this inner world, unique and unrepeatable.

Knowledge clusters are identified by the number of the container node in which they are stored as well as by the polarization value. If a quantum (or a cluster) is to be added to a cluster, a clone of this quantum (cluster) is added to this cluster and no connections or relationships between the sub-tree branches are created. By the way, this is the basis for the functioning of associative thinking. The motive for such an association may be the coincidence (partial or full) of the polarization frequencies of this quantum and root quantum of the cluster under consideration. In the case of cluster association, there is the same condition, but it refers to the polarization frequencies of root quanta of associated clusters. The semantics of an association is determined depending on the context. For example, the term "attack" can be associated with such entities as viral disease, hostilities or animal behavior, and others.

The information stored in memory can be lost (forgotten). This process is somewhat reminiscent of waves that diverge in all directions, caused by an object falling into the water. At the first moment, these waves have a maximum amplitude, but eventually they decay and disappear. Knowledge must be requested, otherwise they are also forgotten, but they do not disappear just like waves on water. For some element of knowledge to disappear, it is necessary to remove the corresponding quanta and clusters from the knowledge tree and deactivate possible references to them in other quanta of knowledge.

Knowledge should be relevant at any time, so they can be changed if necessary. However, knowledge can vary in content, but not in structure. This means that the clusters, being created, are not reconstructed in the future. When an external environment changes, the corresponding clusters of knowledge do not change their structure. Structural updating of knowledge is possible only by complete eliminating existing clusters and creating new ones. After the cluster is eliminated, it is not immediately removed from memory, but remains there for some time until search requests are received and disappears completely if the requests terminate. This is like the procedure for deleting records from a database. Records in the database are also not deleted immediately when the deletion transaction is completed, but only marked as such that it should be deleted.

\subsection{Knowledge Quanta and Thinking}

Knowledge quanta are an active substance that is capable to produce vibrations and thus affected the brain, which in response generates the appropriate commands. The format of these commands includes the action code (or actions) and the identifiers of the participants to which this action refers. If something arises as a result of this action, this something is stored in memory first as a fact, and then - as a quantum of knowledge. Thus, knowledge, as an active substance, affects the brain, and the brain, in turn, can give out commands, as a result of which the knowledge that initiated these commands can be modified. In this way knowledge is self-organized. The ability to self-organizing is their characteristic feature, which makes it possible to distinguish knowledge from ordinary information (not the data) that is passive and simply stored in memory.

To realize the property of self-organization of knowledge quanta, firstly, technologies that would ensure these elements the ability to reproduce themselves, that is, to create their own kind, are needed, and, secondly, they must be intellectual, that is, they must have memory and a mechanism of thinking. Self-organizing elements of knowledge in themselves represent a mechanism of thinking. In other words, thinking is a process of collective interaction of intellectual elements of knowledge that are self-organized, which results to that the new knowledge appears. 


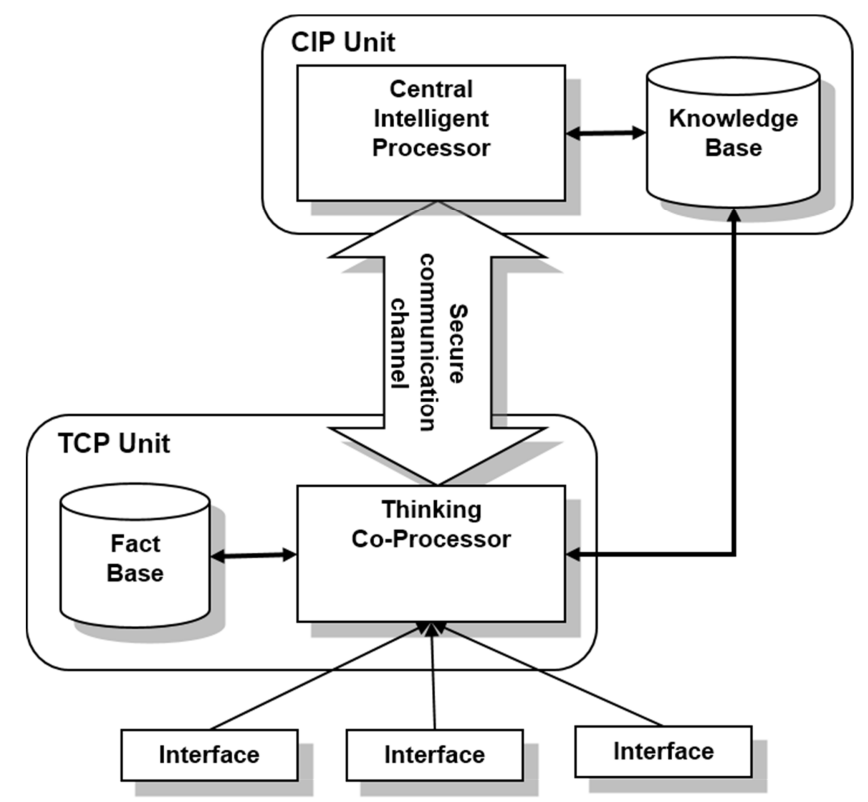

Figure 2. Generalized block diagram of a knowledge-based system with the knowledge self-organizing.

The result of the process of thinking is in the construction of chains of interrelated knowledge quanta (chains of thinking). These chains consist of the quanta with the same polarization, which can be in any of the subtrees of the knowledge tree. The chains of thinking are not clusters, they are created dynamically in memory and disappear as a result of clearing memory when switching consciousness to another problem or simply by switching attention to another object. To explain the process of formation of the chains of thinking, let introduce some abstract knowledge-based system, which consists of two main units: Intelligent Control Centre (Central Intelligent Processor related with the system knowledge base, CIP Unit) and Thinking Control Centre (Thinking Co-Processor related with the system fact base, TCP Unit), as shown in Figure 2.

Knowledge quanta are organized in chains of thinking in the order of decreasing importance for the problem under consideration. The importance of quantum is determined by the sum of the scores they collect for a given task. The calculation of the quantum rating in the chain of thinking is carried out according to a certain algorithm by CIP.

When the signals (visual, verbal, tactile, olfactory) come to the sensory inputs, TCP processes these signals, translating them into the quantum polarization value using a special function (matching function), and sends them to CIP for analysis, whether there is a problem that should be resolved. If CIP fixes the presence of a problem, a command is given to find the quanta associated with this problem and construct a chain of thinking, which just is the solution to the problem (let it call as Problem Solution Chain, PSC).

Building PSC begins in CIP with the definition of a quantum, which is the key to the problem being solved. This quantum plays the role of the signature of the problem: in its memory, the entire chain is stored in the form of a list. By the value of polarization, it is always possible to determine the corresponding input signals using the matching function, which obviously must be reversible and unambiguous. While forming a chain of thinking, two or more quanta may have the same importance. In this case, they line up so that a quantum whose frequency of the polarization vector is closer to the frequencies of the previous quantum lies above. Memory for storing PSC is dynamically allocated and released as the interest in the problem is lost (the problem is forgotten). Thus, PSC is stored in the memory of the key quantum of the problem and is read from there as needed, unless previously lost due to lack of motivation for interest in the problem for a long time.

It is important to note that the PSCs are formed by the knowledge quanta themselves after TCP has determined the key quantum of the problem. Knowledge quanta are divided into two types: first kind quanta have a search processor, second kind quanta have no one. The main task of the search engine is to search for quanta in the knowledge tree with a certain polarization value. When building PSC, firstly quanta of the first kind are searched, they form the framework for solving the problem. Then found quanta of the first type trigger the search for the quanta of the second type which are relevant to this problem and provide a more complete representation of the solution to the problem, supplementing it with semantic shades and nuances.

\subsection{Self-organization of Knowledge}

Knowledge is in a constant movement, they are always active, because namely knowledge provides the survival of the organism, be it either an individual or a society. However, this is possible only if the knowledge is true. It is obvious that false knowledge cannot provide the necessary minimum level of survival. Whether knowledge is true or false depends on their source. If knowledge is obtained from a reliable source, they are true, otherwise - false. It is generally accepted that knowledge gained from experience is true and their source is reliable. Nevertheless, experience is a subjective concept and knowledge obtained by experience is often false, even if they are not contrary to common sense. There is the only reliable source of origin knowledge - the Absolute Fountainhead, all other sources are not reliable.

The criterion of the truth of knowledge can be their success. Knowledge is successful if it allows you to change the environment in favor of the body and thereby increase its life potential. Successful knowledge like sails on a yacht helps to move forward towards the goal. Unsuccessful knowledge, like underwater reefs, can lead to fatal consequences - they are useless.

Knowledge is self-organized as it is accumulated and used, but it should be borne in mind that the property of self-organization is possessed only by true knowledge, and this is a perfectly logical explanation. Knowledge should ensure the correct assessment and analysis of the situation and allow making the right decisions. False knowledge does not allow you to make the right decisions, and this leads to a decrease in the viability of the object that makes such decisions. Such knowledge does not contribute to the survival of the individual in the environment and must be eliminated, 
otherwise, there is a threat to the existence of the individual.

If false knowledge is obtained, corresponding knowledge quanta appear in one or more nodes of the knowledge tree. If false knowledge grows to the size of the whole knowledge tree, then knowledge turns into anti-knowledge. If the knowledge tree is filled with half-true knowledge, it "suffers" in the same way as living trees as a result of pests and diseases.

In order knowledge to self-organize and ensure the adoption of correct decisions, they must satisfy several conditions. First, knowledge quanta should be able to create chains of solutions, and this is ensured by their internal structure, i.e. the presence of a polarization vector and search engine that allows them quickly to find each other in the knowledge tree. Knowledge can be self-organized only because knowledge quanta have a search engine that works according the certain algorithm, which essence is as follows. After determining the key quantum of the problem, it starts searching for knowledge quanta with the same polarization as it does. Then it delegates its search activity to the found knowledge quanta, so the search wave comes into being, and those, in turn, start the same search algorithm, but each one - in its own frequency range.

The second necessary condition for the self-organization of knowledge is overcoming the threshold of redundancy, that is, for an adequate decision the individual must have a certain amount of knowledge, not less than a certain threshold value. Only then one can talk about the rationality of choice in decision-making since rationality is provided by enough knowledge. A person does behave rationally, but only if he has enough knowledge, otherwise the classical models of rational choice turn out to be inapplicable. "The theory of rationality, which does not take into account the complexity of solving problems, is certainly imperfect." [52] This theory is not adequate without the search algorithm for NP-complete problems, but the fact is impossible to create a complete search algorithm in principle, it will certainly be incomplete because of the inevitable abstraction in describing the problem. An alternative to a full search of options could be the model of self-organizing knowledge.

And finally, the third necessary condition for the self-organization of knowledge is to provide access to true knowledge. Every person who has ever faced a choice in the decision-making process is familiar with the situation when, after intensive accumulation of knowledge and their "digestion", the "insight" moment suddenly comes. At such moment consciousness is turned off, and the solution found is called subconscious or heuristic. Usually, this happens in the morning just after waking up or even in a dream. This is not surprising - access to the subconscious is fully possible when only consciousness is turned off, besides that self-organization of knowledge requires a certain amount of time depending on the complexity of the problem being solved. Hence one can conclude that it is the subconscious to be the source of true knowledge, and access to it is needed the knowledge to self-organize. Self-organization of knowledge is a complex and long-term process. It occurs in the brain of a person while sleeping when the consciousness turned off, and "starts up" only if the knowledge reaches a certain "critical mass", which is individual for each person.

As a result of the self-organization of knowledge, new knowledge appears. For example, knowledge about the structure of the brain can generate knowledge about the memory inner structure. Thus, the self-organization of knowledge is, in fact, the establishment of a certain syntax for the procedure for creating knowledge. Knowledge can produce new knowledge due to the ability of knowledge quanta, having a search engine, to recognize each other and form clusters. Knowledge has properties that are inherent only to them and nothing else in the Universe, like living beings it can self-similar reproduce. This is the emergent property of knowledge, which can be called as the idiosyncrasy of the collective interaction of knowledge quanta.

\subsection{Model of Behavioral Act}

In a broad sense, it is customary to understand consciousness as the ability of a person to perceive and cognize the world around him and himself in this world with the help of thinking and his mind. Therefore, the corresponding category of knowledge falls, obviously, into the fourth skeleton subtree in the knowledge tree "Knowledge about the structure of the external environment and its place in it". Awareness of its place in the external environment is essential for humans, because animals also have consciousness, but they do not recognize themselves as part of the world.

The stem of consciousness is a mental activity based on the transformation of signals from the senses to the brain (perceptual act) into an internal representation which closely related to imagination and memory [61]. These signals are converted into impulses of neurons, which in neuroscience are called codes. In accordance with some modern concepts [62, $63]$, neural codes are wave packets, the various frequency and space-time characteristics of which carry information about the stimuli acting on an organ. Each sense organ has its own model of perception and, accordingly, its own set of codes. Thus, we will proceed from that neural codes are packets of codes that are processed in the brain simultaneously (in parallel). The result of this processing is the formation of an environmental image (perceptual image). The mechanisms of the image formation are still poorly understood, but there is a hypothesis that the perceived surrounding space activates a group of neurons ("neural ensemble"), which create a common holistic image of this space, and the images of individual objects are created in accordance with certain neural models of perception (detector model, frequency filtering model, and others) [64, 65].

The formation of images of the surrounding space is ongoing. They consist of many blocks: visual, auditory, tactile, taste, olfactory, spatial-orientational, and are recorded in a special area of memory. This vital behavior-driven memory (perceptual memory) serves to ensure the process of perception and should be assigned to the category I.5 "Life of a biological being", in which the following subcategories can be distinguished: 


\section{I.5.1. Attracting life potential (survival)}

I.5.2. Perceptual memory

\section{I.5.3. Motor memory}

I.5.4. Reproductive function

I.5.5. Communication with the Creator

The volume of perceptual memory compared with the volume of the rest of the memory is obviously small (the most probable value is about 1-2 Tb) and most of it is occupied by visual pictures since visual perception is of particular importance for the life of the body. The corresponding area of perceptual memory (call it perceptual video memory or simply video memory) is constantly reproduced in the internal vision, and, apparently, the pictures are randomly selected. This process continues even in a dream, but in a state of sleep there is no perception and the brain begins to "draw out" pictures or parts of them for reproduction from any of its departments and may even "glance" at the part that can anticipate events in the short term (every person has such an ability, only it differently developed for everyone), and then prophetic dreams arise. Similarly, various representations arise in the inner vision caused by one or another psychoemotional state.

In terms of basic programming algorithms, video memory operates on a queue-by-priority basis, that is, when new pictures arrive in the video memory, the oldest with the lowest priority are reset to the main memory, from where they are deleted over time. At the same time, in addition to perception, sources of newly added pictures can be both imagination and memory. However, some pictures or their details can remain in memory for a rather long time (it can be comparable with the lifetime), since the sensory-emotional state can strongly influence the process of remembering any information (not just visual). For example, if a person is sick, a sense of danger and negative emotions make him perceive much more information and remember it more effectively. The stronger the emotions experienced, the longer the pictures associated with them are stored in memory. Such pictures are stored in the so-called long-term memory, unlike unemotionally linked pictures that are stored in short-term memory and disappear with time. In general, there are areas of short-term and long-term memory in all five skeleton subtrees of knowledge tree, differing from each other in quantum activation codes: some of these codes correspond to short-term memory and the others - to long-term memory.

Simultaneously with the formation of the image of the surrounding space in perceptual memory, control signals are generated in the brain that determine the physical activity of the body. The motor reaction can be both on the whole image, and on its individual elements (selectivity of perception). Selective perception is the basis of the body's life, but according to Miller's law " $7 \pm 2$ ", a person can respond to no more than 7-9 objects simultaneously.

There is every reason to believe that the signals that the brain generates and transmits to muscles are also the wave packets with certain sets of frequencies. For each muscle group, as well as for each organ of perception, there is a certain set of frequencies that are perceived only by these muscles. For each part of the musculoskeletal system, the brain forms its own case of control signals, including packets for certain muscles, and these packets are transmitted through the corresponding channels of the nervous system.

In each muscle, in its head part, there is a nerve node (motor neuron), which plays the role of a controller that controls the work of this muscle. The same node decodes the signals coming from the brain: "reduce", "relax", "degree of reduction/relaxation" and "disconnect".

As the image of environment perception is being formed, the brain simultaneously creates a prototype of the muscle reaction preimage (motor activity) in the given environment, in this situation. This reaction may be immediate or delayed depending on the situation. In any case, the corresponding set of motor operations (motor content) is created and stored in the motor memory (in the knowledge tree this is subcategory "Motor memory" of the category "Life of a biological creature"), from where it can be extracted at any time. Under certain conditions, motor content falls into the area of long-term memory. This way skills are formed.

For example, how does a person learn to dance? At first, his movements are clumsy, constrained, since there are no necessary motor skills in his memory yet, the brain can offer only similar ones. Over time, as he learns the movements become more accurate and coordinated, reaching the level of automatism, that is a person remembers a whole sequence of movements. This can be easily explained if we assume that several motor contents merge into one, which is executed as one movement.

Or the well-known phenomenon of "memory of the hands", when a person cannot remember how to do something, but the hands themselves "remember" the necessary movements. This is possible only if motor skills, or rather physical activity programs and the corresponding operational perception images, are stored in a person's memory and are extracted from there in a problem situation.

And finally, for motor memory, as well as for any kind of memory, forgetting is inherent. If you do not repeat the necessary movements for a long time, they are forgotten, but at the same time, they are restored much faster than during the initial formation due to the preservation of physical activity programs in memory.

Like the image of the environment perception, the corresponding primary prototype of the muscular reaction is created in a certain center of the brain constantly in a state of wakefulness. The formation of such a prototype is an extremely difficult task. Nevertheless, that problem can be solved if there is a virtual model of the motor activity of the body. Today, such models are created using the methods of skeletal 3D animation and the corresponding equipment for capturing movements [66, 67]. Then the task of forming a muscle reaction program can be reduced to the inversing the task of modeling the movement of the corresponding living object.

Repeating movements, such as walking, breathing, blinking, etc., are carried out unconsciously, as if in the background, but according to the same pattern. Some of them turn off in a state of sleep, but others, such as breathing or heart contractions, 
are performed continuously.

There is a consciousness control center in the brain that monitors the current state of perceptual video memory and motor activity and, depending on the situation, turns on one or another motor content or is in standby mode. The commands of this center have the highest priority for the brain, so it plays the role of a supervisor for all other control centers of the brain which act as controllers of various subsystems of the body. The signals entering the brain from various subsystems are naturally processed in parallel, but the corresponding control commands are executed in decreasing order of priority of these subsystems: sensory subsystems (vision, hearing, smell, tactile receptors), musculoskeletal, endocrine, nervous, digestive and excretory. This priority may be violated depending on the level (strength) of the signal. For example, a signal in the form of a strong smell, touching a very hot object or a strong sharp sound signal will be processed first and the corresponding prototype of the muscular reaction will be involved: overlapping (blocking) of the upper respiratory tract, abrupt withdrawal of the limb or turning the head towards the sound source.

As an example of the implementation of the described perceptual act model, we consider a possible scenario for the behavior of a robot meeting an obstacle in its path. First, the robot must identify this obstacle using the video image recognition technique, and then make an appropriate decision. If the robot control unit is an intelligent device, the circuit of which is shown in Figure 2, where CIP is an analog of the consciousness control center, TCP will start the process of searching for a suitable chain of thinking for a fixed problem or it will be created again. After the CIP completes the analysis of the current situation, a suitable prototype of the muscle reaction will be searched in the memory or, otherwise, it will be created by trial and error until the goal is reached. Thus, the robot is trained to find problem solutions based on its own "life experience".

To identify an obstacle, the CIP launches a search and pattern recognition mechanism that finds video clips showing a similar object in the memory and starts the pattern recognition procedure. If the object is recognized, the corresponding motor content is searched, with the help of which the problem situation is resolved. Otherwise (the object is not recognized), a new prototype of motor activity associated with the current image of the environment is created, while another motor contents corresponding to the found video frames with images of objects similar to the current one can be used, in accordance with the principle of analogy (obstacles can be different, but the robot can overcome them in like manner). The final decision is made by the CIP based on the analysis of the current situation, using the decision-making methodology. Like the pattern recognition technique, decision-making techniques are key elements in the model of a behavioral act. The knowledge necessary for this is contained in the subcategory I.5.1 "Attracting life potential" of the knowledge tree:

I.5.1.1. Search engine and pattern recognition

I.5.1.2. Decision-making methodology
I.5.1.3. Adaptation to a changing environment

I.5.1.4. Biological evolution

I.5.1.5. Spiritual enhancement

\section{Metaphysics of Organisational Knowledge Transformation}

\subsection{Explicit and Tacit Knowledge}

Knowledge can be transferred from one individual to another verbally or through symbols. The symbolic way of transferring knowledge is the main way of sharing knowledge among people. Representation of knowledge in the form of a sequence of symbols - this is the formalization of knowledge. Not all knowledge is well formalized, there are poorly formalized knowledge or explicit knowledge as well as knowledge which are not formalized at all or tacit knowledge.

Knowledge is formalized well if it is structured. The structure of knowledge can be defined as a categorical one, that is, such as referring to different categories connected with each other by some of the relations. One can define twelve kinds of relations between the categories of knowledge:

1. relation of subordination (genus-specie),

2. relation of generality (general-individual),

3. relation of aggregation (part-integer),

4. relation of modality (conditionally-unconditionally, possibly-impossibly, necessarily-optionally, etc.)

5. relation of order (more-less, higher-lower, etc.),

6. relation of causality (cause-effect),

7. relation of tactility (degree of semantic proximity),

8. relation of vitality (role in the life of the individual),

9. relation of wisdom (influence on mental abilities in a broad sense),

10. relation of chaos (randomness of the established relationship),

11. relation of variability (the ability to change the categories themselves),

12. relation of effectiveness (ability to generate control instructions).

Some of these relationships, such as subordination, generality, aggregation, and others, are traditionally used to represent knowledge structures in the semantic networks. Other relationships, such as vitality, wisdom, variability or effectiveness, are highlighted as a result of the study of the relationship between the concepts of organizational memory and organizational knowledge structures [60]. These relationships can obviously reflect the nature of the links between node and quantum of knowledge in the individual knowledge tree, although the relationship between the categories of individual and collective knowledge is yet to be explored.

The formalization of knowledge is closely related to consciousness and, accordingly, to the rational behavior of a person. The source of tacit knowledge is the subconscious, it also provides the irrational behavior of a person. The connections between consciousness and explicit knowledge and between the subconscious and tacit knowledge are like 
highways with constant intense movement in both directions (Figure 3). True knowledge is transferred from the subconscious to the tacit knowledge, and in the opposite direction - information about the state of self-organization and the completeness of knowledge. Information about the state of the environment and assessment of the current situation, which is coming from consciousness, forms an explicit knowledge, whereas knowledge of how to behave in the current situation is transferred in the opposite direction.

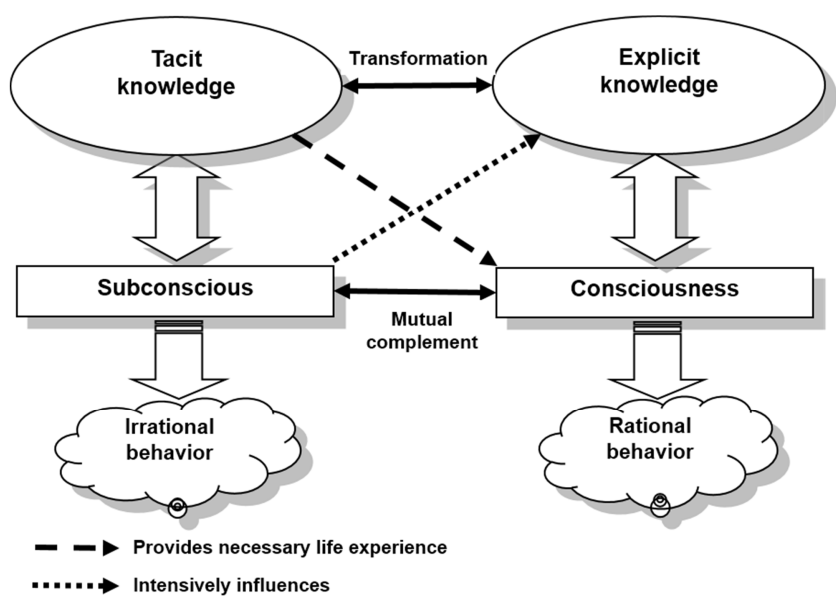

Figure 3. Interaction of explicit and tacit knowledge with consciousness and subconsciousness.

The subconscious and the consciousness complement each other, but they are never used in parallel: if the consciousness is active, the subconscious does not work and vice versa. It is possible to access the subconscious in a state where consciousness is active, but it looks like an off-line request by e-mail.

The relationship between explicit and tacit knowledge can be called a transformation, but the fact is that the transformation of tacit knowledge into explicit knowledge is impossible in the general case. As a rule, tacit knowledge cannot be formalized, except for the development of concepts, hypotheses, models and in the case of obtaining heuristic solutions also. The flow of explicit knowledge into tacit knowledge is a common occurrence, for example, in the development of skills, proficiency, development or identification of abilities for a particular activity, etc.

At the same time, the subconscious has an intensive influence on the formation of explicit knowledge, and tacit knowledge provides the consciousness with life experience. Indeed, let's say a person is actively looking for a solution to a problem. The consciousness "scans" explicit knowledge and, if the solution found, issues it in a formalized form. Otherwise, the stream of thoughts is fixed by the subconscious and, when the consciousness is turned off, the search for relevant tacit knowledge starts or they are created again if the conditions for self-organization are satisfied. When the sought-for knowledge is obtained, it is transferred to the consciousness already in a formalized form.

Let the consciousness fixes the current situation in the environment. If the mind is not able to assess this situation, the problematic "package" of thoughts is transferred to the subconscious, which triggers the search for related knowledge, but this takes time. While the search is performed, the situation can change and then the processing of the next situation is started. So, the tacit knowledge found in the previous situation is saved in the form of life experience and can be used later, if the situation repeats again.

\subsection{Formalization of Organizational Knowledge}

The knowledge contained in the organization's knowledge base must be formalized. The formalization of knowledge is carried out in two stages. At the first stage, knowledge is accumulated in the minds of employees as described above. At the second stage, the knowledge is organized in the form of instructions, technological maps and other official organizational and technical documentation. Procedure of organization of explicit knowledge is similar as the self-organization of individual knowledge, that is, the organization of formal knowledge is also based on categorization and ultimately reduces to building a knowledge tree of the organization or, as it is called, the organization's ontology of knowledge. Explicit knowledge can also be self-organized, for that, obviously, they must be active and satisfy the conditions described above.

The formalization of knowledge means that knowledge must be unified both in structure and in semantics. Unification by structure implies a fixed set of concepts, properties, characteristics and relationships, and unification by semantics is the definition of synonymous representations of the same entities and their types. Unification of knowledge by structure is carried out in the same way as data. Unification of knowledge by semantics is much more difficult since the morphological and syntactic analysis of the elements of knowledge is required.

Knowledge of the individual as well as knowledge of the organization are constantly in motion, they improve, move from one form to another. Explicit knowledge is improved by simply changing its content. Improving tacit knowledge is possible through their formalization, if it is possible, or by improving the relevant skills, which has been developed by the knowledge. That is why the process of developing a corporate culture of knowledge and skills sharing between employees is so important for the organization.

To transmit tacit knowledge that cannot be formalized, special conditions are needed. These conditions are well known and are reduced to providing direct contact of employees, during which they can get from each other information by means of visual observation or verbal discussion of problems which are interesting in both. The effectiveness of such transfer of knowledge depends on many factors both subjective and objective the ability of staff to listen, compare, analyze and draw conclusions, their personal motives and interest in the transfer and improvement of their knowledge.

The effectiveness of the transfer of tacit knowledge through direct contact of staff can be significantly improved if the transfer of knowledge is carried out directly in the 
performance of the duties of the knowledge transfer officer and is accompanied by his comments, and for the perception of this knowledge, the other party uses the method of multi-perception. This method is known in cognitive psychology, but, as a rule, it is used inefficiently. When applying the method of multi-perception, the object of perception is divided into several parts, each of which, in turn, is divided into several segments. All segments are perceived simultaneously, but each segment must be perceived as a separate object. As a rule, this condition is neglected, and segments are perceived as elements of some larger structural units, as a result, the segments lose their "individuality" and their characteristics are unified.

Tacit knowledge that can be used only by their owner and no one else and cannot be transferred to other individuals (authentic knowledge) can be reproduced. To do this, first one can try to formalize this knowledge by observing, communicating with the carrier, analyzing the results of its activities, and then replenishing the missing elements empirically. However, to agree to share his authentic knowledge, an employee must be interested in creating conditions under which the achievement of corporate goals is consistent with his personal benefit. This is also the root cause of the intuitive resistance of employees to the introduction of various automated management systems. The fact is that such systems implicitly contribute to the unification of knowledge, and thus can "pull out" the authentic knowledge of employees.

The procedure of knowledge formalization depends on the way knowledge is presented. The easiest way is to present knowledge in the form of database records. In this case, all knowledge belongs in the same logical and physical structure, and access to them is done through the interface of the selected DBMS. This way of representing knowledge can be used to store facts, but to implement active self-organizing elements of knowledge is not applicable.

Representation of knowledge in the form of logical rules or product rules makes it possible to realize the mechanism of logical inference (pseudo-thinking). However, the effectiveness of these ways of representing knowledge is so low that a machine with intelligence on their basis proves to be more stupid than a cat, which is capable to compare various options for achieving an objective and choose the most optimal one.

Usually, tacit knowledge is transferred from the individual to the individual in the process of direct informal communication. In such communication, first, it is important to create conditions for the self-organization of knowledge for each of the participants. The most appropriate option for organizing such communication is the method of brainstorming when the collocutors can exchange views on a given topic without any restrictions.

Thus, a necessary condition for the transfer of tacit knowledge is the free exchange of knowledge with direct contact of interested parties, and enough condition is the self-organization of their knowledge. It should be noted that for the self-organization of knowledge in the process of their transfer, in addition to the above-mentioned internal conditions, external factors must be provided that can vary significantly depending on the type of knowledge, the level of preparedness of participants and the external environment in which knowledge is transferred. These factors particularly include the following: the motivation of participants to perform their roles, the availability of technology tasks or plans, the availability of common goals and focusing on the result.

"Intelligent people" come to "reasonable" conclusions in circumstances where it is not possible to apply classical models of rational choice. We know little about how they do it" [68]. They turn to their subconscious, are not they? If a person making a decision uses only rational resources of his intellect, he is doomed to be satisfied with not more than the maximization of the expected utility, using rational procedures or models for this. Only a small part of what is hidden in the subconscious passes through the stream of consciousness, so one of the main purposes of the organization is to create conditions to open the channels to obtain knowledge through the subconscious of its employees. Nonaka and Takeuchi defined the conditions as follows [58]:

1. existence of an organizational intention, that is, the organization's desire for the set goal;

2. independence (or autonomy) of the actions of individuals;

3. shake-up and creative (deterministic) chaos which is necessary for creating structures of self-organization and transfer to a new order in the organization;

4. redundancy of information, stimulating the exchange of tacit knowledge among employees;

5. diversity of information within the organization.

\subsection{Process of Organizational Knowledge Creation}

Each new loop of the SECI knowledge creation spiral is formed as the chain:

$<$ distribution of non-formalized knowledge $>$

$<$ concept $>$

$<$ check $>$

$<$ archetype $>$

$<$ transition to the next level $>$,

followed by the transition to a higher organizational level from subjective up to inter-organizational. Paying tribute to the authors of the SECI model, which analyzed in detail all possible transitions of tacit and explicit knowledge that really represent the essence of the process of knowledge transformation, let us make some critical remarks. First, it should be noted that the analysis of the transformation of organizational knowledge is carried out without considering the inner properties of the knowledge itself and the state this knowledge is in, that is, its level of self-organization. This is also important because the criteria for transition to a higher ontological level are not clearly defined. In this regard, we note that both tacit and explicit knowledge can be in a different state of order - from complete order to complete chaos, and these conditions differ for the levels of individual and collective knowledge. The transition to the next level spiral of knowledge is possible only under the condition that 
there has been a certain level of order and the formalization degree of knowledge which are adequate in terms of the objective (or sub-objective). If this state is not achieved, the transition to the next level will be premature and finish most likely by returning to the previous or even lower level of the knowledge creation spiral.

Each new loop of the knowledge creation spiral begins with the distribution of information in order to create redundancy in the organization. Then the process goes to the stage of deterministic chaos, which is often created artificially to support the actual distribution of tacit knowledge. For the level of individual knowledge, this is natural. At this level, disparate elements of tacit knowledge are clustering, resulting in ideas, guesses, hypotheses, that is, tacit knowledge is partially formalized. Further, the formed cluster of new knowledge begins its movement (verification) from the unordered and poorly formalized state, seeking to reach a certain threshold of formalization. This is how the working concept is formed. If this threshold is not reached up to the end of the spiral loop, the cluster passes to the next loop or even to the previous loop, but not to the next organizational level.

Thus, the process of organizational knowledge creation is certainly spiraling, but with possible backward returns to previous levels of the spiral if necessary. This process is consistent and progressive: tacit knowledge of employees goes through the stage of self-organization at the level of individual knowledge, partially being formalized, and moves to the level of working groups in the form of a concept, that is, to the next ontological level. At the level, poorly formalized conceptual knowledge passes through the same stages and turns into an archetype, which is ultimately brought to the level of finally formalized and fully ready to use the collective knowledge of the organization. In this way, here is the development of the idea of SECI knowledge creation model on the basis of the concept of self-organizing knowledge.

All things considered, let us note three key, in our opinion, moment for understanding the metaphysics of the transformation of knowledge in an organization: firstly, it is the primacy of the knowledge of individuals, which purposeful synergistic interaction results in collective knowledge creation and makes necessary conditions for the development of an organizational knowledge spiral in space and time; secondly, the mechanism of the mutual knowledge transformation between tacit and explicit knowledge is based on the native activity of knowledge and their capacity for self-organizing; thirdly, an organization should be viewed as an open evolving system, passing in its development the stages of organizational shake-up and creative chaos without which innovation is impossible.

\section{Discussion and Conclusion}

It should be noted that "full-stack" developing KM framework includes a set of tasks having to be decided: development of a knowledge representation model, clarifying the metaphysics of transformation process of both individual and collective knowledge, development of methods for structuring knowledge, forming requirements for the formalism of representation and manipulation of knowledge and the choice (or development) of an appropriate programming system that implements this formalism, creating a workflow infrastructure. Only some of them that lay the foundation for further research are presented in the article. To sum up, a conceptual model of knowledge representation as an active intellectual substance, which has emergent self-organization property resulted from the idiosyncrasy of collective interaction of atomic structural elements (knowledge quanta), is developed. The forces of collective interaction of knowledge quanta are aimed at ensuring the survival of the organism in the external environment and determine the formation of hierarchical knowledge structures in the memory. As a result, knowledge constantly generates new knowledge due to its ability to be self-organized that is essentially the basis of the mechanism of thinking.

Creating new organizational knowledge is a complex process of mutual transformation of tacit and explicit knowledge. For individual knowledge, this process is affected by the natural interaction of consciousness and subconsciousness. For collective knowledge, certain conditions should be provided that promote, firstly, self-organization and formalization of tacit knowledge of employees and, secondly, the exchange of such knowledge among employees. To do this, the KM system in the organization should play the role of an intersubjective environment for a purposeful dialogue between employees of the company, contributing to the creation of new knowledge and value chains.

Considering knowledge as an active substance with the emergent ability to self-organize and self-reproduce, it is certainly possible to solve the key urgent problems of building the KM framework in organizations, so that such terms as organizational memory, organizational wisdom or mind gain quite a definite meaning and interpretation. The proposed approach to the development of the organizational KM system makes it possible to critically approach the SECI model by Nonaka and Takeuchi, complementing it with two scopes that describe the degree of self-organization and the state of knowledge ordering. Moreover, it makes reconsider the role of the KM system so that it becomes an adaptive environment for conducting and interchanging of knowledge. This evolution of KMS may be called Knowledge Driven System [69].

The issue of the knowledge categorization is affected only to the extent necessary for the completeness of consideration of the proposed model, and it is the subject for peculiar study.

\section{References}

[1] Searle, John Rogers (1984). Minds, brains and science: The 1984 Reith lectures. Cambridge, MA: Harvard University Press.

[2] Luger, George F. (2009). Artificial intelligence: Structures and strategies for complex problem solving (6th ed.). Boston, MA: Pearson Education, Inc. 
[3] Chen, Serena H., Jakeman, Anthony J., Norton, John P. (2008). Artificial Intelligence techniques: An introduction to their use for modelling environmental systems. Mathematics and Computers in Simulation, 78, 379-400.

[4] Corea, Francesco (2018). AI Knowledge Map: How To Classify AI Technologies. Retrieved from: https://www.forbes.com/sites/cognitiveworld/2018/08/22/ai-k nowledge-map-how-to-classify-ai-technologies/\#60792f40777 3.

[5] McKinsey Global Institute (2018). The promise and challenge of the age of artificial intelligence. Briefing note prepared for the Tallinn digital summit October 2018. Retrieved from: https://www.mckinsey.com/featured-insights/artificial-intellig ence/the-promise-and-challenge-of-the-age-of-artificial-intelli gence.

[6] Zadeh, Lotfi A. (1994). Fuzzy Logic, Neural Networks, and Soft Computing. Communications of the ACM, 37 (3), 77-84.

[7] Ding, S., Li, H., Su, C. et al. (2013). Evolutionary artificial neural networks: a review. Artificial Intelligence Review, 39, 251-260.

[8] Xin Yao, Senior Member, IEEE, and Yong Liu (1997). A New Evolutionary System for Evolving Artificial Neural Networks. IEEE Transactions on Neural Networks, 8 (3), 694-713.

[9] Xin Yao (1993). A Review of Evolutionary Artificial Neural Networks. International Journal of Intelligent Systems, 4, 539-567.

[10] Mnih, V., Kavukcuoglu, K., Silver, D. et al. (2015). Human-level control through deep reinforcement learning. Nature, 518, 529-533.

[11] Mnih, Volodymyr, Kavukcuoglu, Koray, Silver, David, Graves, Alex, Antonoglou, Ioannis, Wierstra, Daan, Riedmiller, Martin (2013). Playing Atari with Deep Reinforcement Learning. arXiv: 1312.5602. Retrieved from: https://arxiv.org/abs/1312.5602.

[12] Nguyen, Anh, Yosinski, Jason, Clune, Jeff (2015). Deep Neural Networks Are Easily Fooled: High Confidence Predictions for Unrecognizable Images. The IEEE Conference on Computer Vision and Pattern Recognition (CVPR), 427-436.

[13] Elbeltagi, Emad, Hegazy, Tarek, Grierson, Donald (2005). Comparison among five evolutionary-based optimization algorithms. Advanced Engineering Informatics, 19 (1), 43-53.

[14] Long, Qiang, Wu, Changzhi, Huang, Tingwen, Wang, Xiangyu (2015). A genetic algorithm for unconstrained multi-objective optimization. Swarm and Evolutionary Computation, 22, 1-14.

[15] Richter-von Hagen, Cornelia, Ratz, Dietmar, Povalej, Roman (2005). Towards Self-Organizing Knowledge Intensive Processes. Journal of Universal Knowledge Management, 0 (2), 148-169.

[16] Chen, Zhen-Yao, Kuo, R. J., Hu, Tung-Lai (2016). An integrated hybrid algorithm based on nature inspired evolutionary for radial basis function neural network learning. International Journal on Artificial Intelligence Tools, 25 (2), 1650004 (25 pages).

[17] Chi-Keong Goh, Eu-Jin Teoh, Kay Chen Tan (2008). Hybrid Multiobjective Evolutionary Design for Artificial Neural Networks. IEEE Transactions on Neural Networks, 19 (9), $1531-1548$.
[18] Christou, Vasileios; Tsipouras, Markos G.; Giannakeas, Nikolaos; Tzallas, Alexandros T.; Brown, Gavin (2019). Hybrid extreme learning machine approach for heterogeneous neural networks. Neurocomputing, 361, 137-150.

[19] Marghny, Mohamed (2011). Rules extraction from constructively trained neural networks based on genetic algorithms. Neurocomputing, 74 (17), 3180-3192.

[20] Venkatesan, D., Kannan, K., Saravanan, R. (2009). A genetic algorithm-based artificial neural network model for the optimization of machining processes. Neural Computing \& Applications., 18 (2), 135-140.

[21] Yedjour, Dounia; Benyettou, Abdelkader; Yedjour, Hayat (2018). Symbolic interpretation of artificial neural networks using genetic algorithms. Turkish Journal of Electrical Engineering \& Computer Sciences, 26 (5), 2465-2475.

[22] Prigogine I., Stengers I. (1984). Order out of chaos: Man's new dialogue with nature. London, UK: Heinemann.

[23] Gutierrez-Navarro, Daniel and Lopez-Aguayo, Servando. Solving ordinary differential equations using genetic algorithms and the Taylor series matrix method (2018). Journal of Physics Communications, 2 (11). Retrieved from: https://iopscience.iop.org/article/10.1088/2399-6528/aaedd2/p df.

[24] Raja Muhammad Asif Zahoor, Khan, Junaid Ali, Qureshi I. M. (2019) Evolutionary Computational Intelligence in Solving the Fractional Differential Equations. 11th Asian Conference, ACIIDS 2019, Yogyakarta, Indonesia, April 8-11, 2019, Proceedings, Part I, 231-240.

[25] Tsoulos, Ioannis G, Lagaris, Isaac E. (2006). Solving differential equations with genetic programming. Genetic Programming and Evolvable Machines, 7 (1), 33-54.

[26] Tsoulos, Ioannis G., Gavrilis, Dimitris, Glavas, Euripidis (2009). Solving differential equations with constructed neural networks. Neurocomputing, 72 (10-12), 2385-2391.

[27] Criado, N. (2013). Using norms to control open multi-agent systems. AI Communications, 26 (3), 317-318.

[28] Metcalf, Lynn; Askay, David A.; Rosenberg, Louis B. (2019). Keeping Humans in the Loop: Pooling Knowledge through Artificial Swarm Intelligence to Improve Business Decision Making. California Management Review, 61 (4), 84-109.

[29] Hosny Ahmed Abbas, Samir Ibrahim Shaheen, Mohammed Hussein Amin. Organization of Multi-Agent Systems: An Overview (2015). International Journal of Intelligent Information Systems, 4 (3), 46-57.

[30] Zou, Yuanyuan; Su, Xu; Li, Shaoyuan; Niu, Yugang; Li, Dewei (2019). Event-triggered distributed predictive control for asynchronous coordination of multi-agent systems. Automatica, 99, 92-98.

[31] Wang, Xiangke; Zeng, Zhiwen; Cong, Yirui (2016). Multi-agent distributed coordination control: Developments and directions via graph viewpoint. Neurocomputing, 199, 204-218.

[32] Barbosa, José, Leitão, Paulo, Adam Emmanuel, Trentesaux, Damien (2015). Dynamic self-organization in holonic multi-agent manufacturing systems: The ADACOR evolution. Computers in Industry, 66, 99-111. 
[33] Leitão P. (2013) Towards Self-organized Service-Oriented Multi-agent Systems. In: Borangiu T., Thomas A., Trentesaux D. (eds). Service Orientation in Holonic and Multi Agent Manufacturing and Robotics. Studies in Computational Intelligence, vol 472. Berlin, DE: Springer-Verlag Berlin Heidelberg.

[34] Rodriguez S., Gaud N., Hilaire V., Galland S., Koukam A. (2007) An Analysis and Design Concept for Self-organization in Holonic Multi-agent Systems. In: Brueckner S. A., Hassas S., Jelasity M., Yamins D. (eds) Engineering Self-Organising Systems. ESOA 2006. Lecture Notes in Computer Science, vol 4335. Springer, Berlin, Heidelberg.

[35] Unland R. (2003) A Holonic Multi-agent System for Robust, Flexible, and Reliable Medical Diagnosis. In: Meersman R., Tari Z. (eds) On The Move to Meaningful Internet Systems 2003: OTM 2003 Workshops. OTM 2003. Lecture Notes in Computer Science, vol 2889. Springer, Berlin, Heidelberg.

[36] Johnson, Norman L. (1998). Collective Problem Solving: Functionality beyond the Individual. Special Issue on Simulation of Social Agents. Kerstin Dautenhahn, Editor. Los Alamos National Laboratory, USA. Retrieved from: https://www.uni-marburg.de/fb12/arbeitsgruppen/datenbionik/ pdf/pubs/1999/ultsch99data.

[37] Hanser, T., Barber, C., Rosser, E., Vessey, J. D., Webb, S. J., \& Werner, S. (2014). Self organising hypothesis networks: a new approach for representing and structuring SAR knowledge. Journal of cheminformatics, 6, 21. Retrieved from: https://www.ncbi.nlm.nih.gov/pmc/articles/PMC4048587/.

[38] Minsky M. (1985). The Society of Mind. New York, US: Simon \& Schuster Inc.

[39] Wagner William P. (2017). Trends in expert system development: A longitudinal content analysis of over thirty years of expert system case studies. Expert Systems with Applications, 76, 85-96.

[40] Sahin, S., Tolun M. R. Hassanpour R. (2012). Hybrid expert systems: A survey of current approaches and applications. Expert Systems with Applications, 39 (4), 4609-4617.

[41] Tong-Seng Quah, Chew-Lim Tan, Krishnamurthy S. Raman, Bobby Srinivasan (1996). Towards integrating rule-based expert systems and neural networks. Decision Support Systems, 17 (2), 99-118.

[42] Yoon, Youngohc, Guimaraes, Tor, Swales, George (1994). Integrating artificial neural networks with rule-based expert systems. Decision Support Systems, 11 (5), 497-507.

[43] Sowa J. F. (ed.) (1991). Principles of Semantic Networks. Explorations in the Representation of Knowledge. San Mateo, CA: Morgan Kaufmann.

[44] Berners-Lee, Tim (1998). Semantic Web Road map. Retrieved from: https://www.w3.org/DesignIssues/Semantic.html.

[45] Brewster C.; O'Hara K. (2004). Knowledge representation with ontologies: the present and future. IEEE Intelligent Systems, 19 (1), 72-81.

[46] Minsky, M. (1975). A Framework for Representing Knowledge. In: P. H. Winston (ed.). The Psychology of Computer Vision. New York, US: McGraw Hill.

[47] Yen, John; Neches, Robert; MacGregor, Robert (1989).
Classification-Based Programming: A Deep Integration of Frames and Rules. DTIC ADA211279. Retrieved from: https://apps.dtic.mil/docs/citations/ADA211279.

[48] Console, L., Rossi, G. (1989). Using Prolog for building frog, a hybrid knowledge representation system. New Generation Computing, 6 (4), 361-388.

[49] Kohonen R. (2001). Self-Organizing Maps /R. Kohonen. - 3 ed. Berlin, DE: Springer-Verlag Berlin Heidelberg.

[50] Ultsch, A. (1999). Data Mining and Knowledge Discovery with Emergent Self-Organizing Feature Maps for Multivariate Time Series. Retrieved from: https://www.uni-marburg.de/fb12/arbeitsgruppen/datenbionik/ pdf/pubs/1999/ultsch99data.

[51] Newell A, Simon H. A. (1972). Human problem solving. Englewood Cliffs, NJ: Prentice-Hall.

[52] Rajeswari P. V. N., Prasad T. V. (2012) Hybrid Systems for Knowledge Representation in Artificial Intelligence. International Journal of Advanced Research in Artificial Intelligence, 1 (8), 31-36.

[53] Bundy A., Wallen L. (1984). KL-One/KL-Two. In: Bundy A., Wallen L. (eds) Catalogue of Artificial Intelligence Tools. Symbolic Computation (Artificial Intelligence). Springer, Berlin, Heidelberg.

[54] Sowa, J. F. (2000). Knowledge Representation: Logical, Philosophical, and Computational Foundations. Pacific Grove, CA: Brooks/Cole Publishing Co.

[55] Serenko, Alexander (2013). Meta-analysis of scientometric research of knowledge management: Discovering the identity of the discipline. Journal of Knowledge Management, 17 (5), 773-812.

[56] Fteimi, Nora (2015). Analyzing the literature on knowledge management frameworks: Towards a normative knowledge management classification schema. ECIS 2015 Completed Research Papers. Paper 51. Retrieved from: http://aisel.aisnet.org/ecis2015_cr/51/.

[57] Holsapple, C. W., Joshi, K. D. (1999). Description and analysis of existing knowledge management Frameworks. HICSS '99 Proceedings of the Thirty-Second Annual Hawaii International Conference on System Sciences, V. 1, 1072. Retrieved from: http://citeseerx.ist.psu.edu/viewdoc/download?doi=10.1.1.99. $3050 \&$ rep $=$ rep $1 \&$ type $=$ pdf.

[58] Nonaka, Ikudjiro and Takeuchi, Hirotaka (1995). The knowledge creating company: How Japanese companies create the dynamics of Innovation. New York, NY: Oxford University Press.

[59] Hodkinson, Gerard P., Sparrow, Paul R. (2002). Competent organization: Psychological analysis of the process of strategic management. Buckingham, UK: Open University Press.

[60] Lyles, Marjorie A., Schwenk, Charles R. (2007). Top management, strategy and organizational knowledge structures. Journal of Management Studies, 29 (2), 155-174.

[61] Kind, A. (2018). How imagination gives rise to knowledge. In: F. Macpherson \& F. Dorsch (Eds). Perceptual Imagination and Perceptual Memory. Oxford, UK: Oxford University Press.

[62] Kashchenko, Serguey (2015). Models of Wave Memory. Cham, Switzerland: Springer International Publishing AG. 
[63] Lebedev A. N., Myshkin I. Yu., Mayorov V. V. (1990). The wave model of memory. In: Holden A. V., Kryukov V. I. (eds.) Neurocomputers and attention. Vol. 1. Neurobiology, synchronization and chaos, 53-59. Manchester, UK: Manchester University Press.

[64] Bekhtereva, N. P. (1978). The neurophysiological aspects of human mental activity (2d ed.). New York: Oxford University Press.

[65] Hubel, David H. and Wiesel, Torsten N. (2005). Brain and visual perception: the story of a 25 -year collaboration. Oxford, UK: Oxford University Press.

[66] Borodulina A. (2019) Application of 3D human pose estimation for motion capture and character animation. University of Oulu, Degree Program in Computer Science and Engineering. Master's thesis, 57 p. Retrieved from: http://jultika.oulu.fi/files/nbnfioulu-201906262670.pdf.
[67] Raut Smita, Kokare Supriya, Shere Sonali, Bansode Priyanka, Prof. J. N. Ekatpure. (2016) Animation of 3D Human Model Using Markerless Motion Capture. International Journal for Research in Applied Science \& Engineering Technology, 4 (VIII), 210-216. Retrieved from: https://www.ijraset.com/fileserve.php?FID=4646.

[68] Simon, Herbert A. (1978). Rationality as process and as product of thought. Richard T. Ely Lecture. American Economic Review, 68 (2), 1-16.

[69] Moroz, O. From knowledge management system to knowledge driven system. MHTK "ABIA", North America, Apr. 2019. Retrieved from: http://conference.nau.edu.ua/index.php/AVIA/AVIA2019/pape r/view/6082/4717. 